\title{
The Prevalence of Incidental Findings in Multiple Sclerosis Patients
}

\author{
J. Trufyn, M.D. Hill, J.N. Scott, J. Modi, V. Ciura, R. Frayne, M. Goyal, D. \\ Lautner, D. Bhayana, W.J. Davenport, J.K. Mah, J.M. Burton, F. Costello
}

\begin{abstract}
Background: Incidental findings arising from imaging research have important implications for patient safety. Magnetic resonance imaging is widespread in multiple sclerosis (MS) studies and care, yet the prevalence rate of incidental findings in MS is poorly defined. The absence of such reports in the MS literature suggests that such findings may be deemed inappropriate for documentation in research publications, or possibly, not fully reported at all. Objective: We sought to document incidental findings from a study designed to detect features of chronic cerebrospinal venous insufficiency (CCSVI) in MS patients and control subjects. Methods: Magnetic resonance images were obtained as part of a prospective study conducted between October 2010 and September 2012. Patients with MS (relapsing-remitting, primary progressive, secondary progressive), clinically isolated syndromes, and neuromyelitis optica and age/sex-matched healthy controls were included. All images were reviewed by neuro-radiologists for quality-control purposes. Results: Magnetic resonance imaging was successfully obtained in 166 participants $(110$ patients, 56 controls $)$. Incidental abnormalities $(\mathrm{n}=33$ ) were detected in $15 \%$ of patients $(n=17)$ and $27 \%$ of controls $(n=15)$, comprising $19 \%$ overall $(n=32)$. Conclusions: The prevalence of incidental findings from the MS population was not significantly different from the control population. However, the overall prevalence was high and warrants a careful management strategy for future imaging studies.
\end{abstract}

RÉSUMÉ: Prévalence des découvertes fortuites chez les patients atteints de sclérose en plaques. Contexte : Les découvertes fortuites lors de l'imagerie faite dans un but de recherche ont des implications importantes pour la sécurité des patients. L'imagerie par résonance magnétique est courante dans les études et les soins dans la sclérose en plaques (SP), et pourtant le taux de prévalence des découvertes fortuites dans la SP est mal connu. L'absence de publications à ce sujet dans la littérature sur la SP suggère qu'on considère peut-être qu'il n'est pas approprié de les inclure lors de la publication des résultats de la recherche ou elles ne sont pas rapportées complètement. Objectif : Nous avons documenté les découvertes fortuites au cours d'une étude dont le but était de détecter des manifestations de l'insuffisance veineuse cérébrospinale chronique chez des patients atteints de SP et des sujets témoins. Méthode : L'imagerie par résonance magnétique a été obtenue au cours d'une étude prospective effectuée entre octobre 2010 et septembre 2012. Les patients atteints de SP (forme cyclique, progressive primaire, secondaire progressive), de syndromes isolés au point de vue clinique ainsi que de neuromyélite optique et des sujets témoins en bonne santé appariés pour l'âge et le sexe ont été inclus dans l'étude. Toutes les images ont été examinées par des neuroradiologistes à des fins de contrôle de la qualité. Résultats : l'IRM de 166 participants (110 patients et 56 témoins) a été étudiée. Des anomalies découvertes fortuitement $(\mathrm{n}=33)$ ont été détectées chez $15 \%$ des patients $(\mathrm{n}=17)$ et chez $27 \%$ des témoins $(\mathrm{n}=15)$, soit au total chez $19 \%$ des participants $(\mathrm{n}=32)$. Conclusions : La prévalence de découvertes fortuites dans le groupe de patients atteints de SP n'était pas significativement différente de celle du groupe témoin. Cependant, la prévalence globale était élevée et justifie une stratégie de gestion prudente lors d'études d'imagerie futures.

Can J Neurol Sci. 2014; 41: 49-52

In Canada, the Tri-Council Policy Statement: Ethical Conduct for Research Involving Humans (2010) states that, "Researchers have an obligation to disclose to the participant any material incidental findings discovered in the course of research." Reporting and managing incidental findings arising from imaging research has important implications for patient safety and it should be integral to the informed consent, image acquisition, and assessment process. Yet, this is often not the case. Among 43 consent forms for magnetic resonance (MR) studies that were approved by Canadian research ethics boards, only $29(67 \%)$ addressed the possibility of unanticipated discoveries. ${ }^{1}$

In studies of multiple sclerosis (MS), MR imaging is frequently employed for both diagnosis and as a surrogate outcome measure, yet the prevalence rate of radiological incidental findings is not well established. Previous studies not specific to MS have detected incidental findings in $14 \%$ to $47 \%$ of MR scans, of which $15 \%$ to $20 \%$ required further referral for appropriate management. ${ }^{2-5}$ We sought to document MR incidental findings from a prospective study designed to detect and compare features of chronic cerebrospinal venous insufficiency (CCSVI) in MS, clinically isolated syndromes (CIS), and neuromyelitis optica (NMO) patients relative to control subjects.

From the Departments of Clinical Neurosciences (JT, MDH, JNS, RF, MG, WJD, JMB, FC), Radiology (MDH, JNS, JM, RF, MG), Community Health Services (MDH), Medical Genetics (WJD), Surgery (Ophthalmology) (FC), Hotchkiss Brain Institute, Faculty of Medicine, University of Calgary; Department of Diagnostic Imaging (DL, DB) and Seaman Family MR Research Centre (RF), Foothills Medical Centre; Department of Pediatrics (JKM), Alberta Children's Hospital, Calgary, Alberta, Canada; Department of Neuroradiology (VC), Massachusetts General Hospital, Boston, Massachusetts, USA.

Received April 8, 2013. Final Revisions Submitted August 27, 2013. Correspondence to: Fiona Costello, Departments of Clinical Neurosciences and Surgery (Ophthalmology); University of Calgary, Foothills Medical Centre; Area 3, UCMC Health Sciences Centre 3350 Hospital Drive; Calgary, Alberta, T2N 4N1, Canada. Email: fiona.costello@albertahealthservices.ca. 


\section{METHODS}

Magnetic resonance images were obtained between October 2010 and September 2012 in a cross-sectional study that aimed to compare the proportion of venous outflow obstructions indicative of CCSVI between MS patients and controls. This study was approved by the University of Calgary Conjoint Health Research Ethics Board (CHREB). Patients included had a clinical diagnosis of MS (relapsing-remitting MS, RRMS; primary progressive MS, PPMS; secondary progressive MS, SPMS), CIS, or NMO. ${ }^{6}$ Participants were excluded if they displayed any of the following: current pregnancy; prior history of jugular or subclavian central line insertion; prior history of chemotherapy, radiation therapy or stem cell transplantation; ongoing use of anti-coagulation or known history of thrombophilia or vasculopathy; any known contraindications to MR imaging including a cardiac pacemaker, cochlear implant, weight greater than $125 \mathrm{~kg}$, or severe claustrophobia; any condition or situation that would put the participant at risk of worsening health if enrolled in the study; any condition or situation that would prevent completion of the study; or were unable to provide written informed consent. Additionally, control subjects were excluded if they displayed any diagnosed neurological condition, were not matched by sex and decile of age for two patient cases, or had a first-degree relative with MS.

All MR studies were systematically reviewed by a neuroradiologist for the presence of incidental abnormalities unrelated to an underlying diagnosis of MS or indicative of CCSVI. Anticipated findings included underlying neoplasms, strokes, vascular malformations, congenital malformations, and/or manifestations of remote trauma. The presence of white matter lesions was not viewed as a noteworthy incidental finding because such lesions are common in MS patients, but can also be detected in normal individuals as part of the normal aging process. $^{7}$ Detection and analysis of features of CCSVI was performed at a later date. Quality concerns were communicated to MR technologists in an effort to improve future images and all incidental findings were relayed to the primary clinical investigator (FC). Any finding that was not previously revealed to us by the participant or documented in the patient's medical chart, but had a potential health implication was conveyed to the participant by telephone or in person. Once this communication was made with the participant, appropriate follow-up and/or health care referrals were arranged on his or her behalf by the principal investigator. Letters documenting the incidental findings were sent to the primary care physicians of each participant with the recommendation for follow-up testing, such as a dedicated thyroid ultrasound in the case of an incidental thyroid nodule. For study participants who lacked primary care physicians, the appropriate investigations and/or referrals were arranged by the primary clinical investigator, who took on the responsibility for their care. For each and every participant with an incidental finding, we generated a formal record of their medical issue, and clinical care path, so that non-study personnel would have access to results.

Our consent process included a discussion on how incidental findings would be managed. Patients and controls were informed that they would be made aware of any observed abnormality of potential health concern. We did not explicitly explain the possible implications of incidental findings (e.g., how insurance could be affected if a serious finding was detected) with participants, prior to their consent for participation in the study.

Magnetic resonance imaging was performed on a $3 \mathrm{~T} \mathrm{MR}$ scanner (Discovery 750; General Electric Healthcare, Waukesha, WI) using a customized neurovascular imaging protocol optimized for CCSVI. The protocol consisted of localizer sequences (head and neck) plus oblique axial 2D fluid attenuate inversion recovery (FLAIR), oblique axial 2D fast relaxation fast spin echo sequence (FRFSE), axial 3D T2*-weighted angiography (SWAN; General Electric Healthcare), sagittal 3D time-resolved imaging of contrast kinetics (3D TRICKS; General Electric Healthcare), sagittal spoiled-gradient echo (FSPGR), and phase contrast (PC) imaging. A summary of the key acquisition parameters can be found in the Supplemental Table.

\section{Statistical Methods}

Data are reported using standard descriptive statistics. To compare the prevalence of incidental findings between patients and controls, Fisher's exact test was used. All tests were twotailed and conventional levels of statistical significance $(\mathrm{p}<$ 0.05 ) were considered relevant.

\section{RESULTS}

Of the 197 participants who provided informed consent at screening, nine withdrew consent and eight were excluded because data became available after the consent process that resulted in an exclusion criterion being fulfilled. This resulted in a final study group of 120 patients and 60 controls. Magnetic resonance imaging was successfully obtained in 166 participants (110 patients, 56 controls). Incidental abnormalities were detected in $19 \%$ of the subjects $(n=32 ; 17$ patients and 15 controls) for a total of 33 findings (Table). These findings included: a cervical spine neural foraminal stenosis (one control), a remote posterior inferior cerebellar artery (PICA) territory stroke (one control), intracranial aneurysms (two patients), cavernomas (five patients, two controls), a cervical spine disc protrusion (one patient), enlarged cervical lymph nodes (one patient), meningiomas (one patient, two controls), a brain parenchymal cyst (one control), pituitary gland structural irregularities (one patient, one control), and thyroid nodules (seven patients, seven controls). One patient had two incidental findings: an aneurysm and a brain parenchymal cavernoma. Of the 32 subjects with incidental findings, $84 \%$ (27) required further investigations, which included follow-up communication in person or by telephone and subsequent referral for other medical services.

\section{Discussion}

The prevalence of incidental findings was not significantly different in MS patients (15\%) relative to the control population $(27 \% ; \mathrm{p}=0.097)$ in our study. However, the occurrence of incidental findings was frequent enough that establishing a process to manage their discovery was both useful and important. Thyroid nodules or cysts were the most common detected abnormality (6\% of patients and $13 \%$ of controls). The high prevalence of thyroid lesions was not surprising, given that thyroid nodules are detected in neck imaging studies performed for other indications in $10 \%$ to $60 \%$ of the general population..$^{8,9}$ 
Table: Magnetic resonance imaging incidental findings in patients and controls

\begin{tabular}{ccc}
\hline & $\begin{array}{c}\text { Patients } \\
(\mathrm{n}=110)\end{array}$ & $\begin{array}{c}\text { Controls } \\
(\mathrm{n}=56)\end{array}$ \\
\hline Mean age, years (SD) & $46(12)$ & $45(12)$ \\
Percent females, \% (n) & $76(84)$ & $73(41)$ \\
Percent with incidental findings, \% (n)* & $15(17)$ & $27(15)$ \\
Total brain incidental findings & 9 & 6 \\
PICA-territory stroke & 0 & 1 \\
intracranial aneurysm & 2 & 0 \\
cavernoma & 5 & 2 \\
frontal meningioma & 1 & 1 \\
parenchymal cyst & 0 & 1 \\
pituitary irregularity & 1 & 1 \\
cervical foraminal stenosis & 9 & 9 \\
enlarged cervical lymph nodes & 0 & 1 \\
spinal meningioma & 1 & 1 \\
disc protrusion & 0 & 0 \\
thyroid nodule/cyst & 1 & 7 \\
\hline
\end{tabular}

*Thirty-three incidental findings in 32 subjects

Cavernomas were the most prevalent incidental finding detected with brain MR imaging.

There are a lack of standardized guidelines for detecting and communicating incidental findings in imaging research. ${ }^{10,11}$ This may explain why one patient in our study was made aware of an incidental finding for the first time, despite the fact that she had participated in numerous CHREB-approved clinical research studies involving MR imaging at our institution. While the members of the Working Group on Incidental Findings in Brain Imaging Research are divided as to whether or not clinical assessment of an imaging study is required, ${ }^{12}$ most MS clinical trials have a neuro-radiologist on the research team. Arguably, establishing a process for the detection, documentation, notification, and management of incidental findings can be labor-intensive. Yet, respecting the rights, safety, and well-being of research subjects is the cornerstone of ethical research. If incidental findings are both anticipated, and then documented and appropriately managed, reporting should be feasible. However, there is a lack of perceived need to report incidental findings and their management as part of the primary analysis of most imaging research studies.

There are ethical complexities surrounding incidental imaging findings, which are well described conceptually in the epidemiological screening literature. ${ }^{13,14}$ Screening for asymptomatic, but early stage disease, has been proposed as a method to reduce mortality from many illnesses, including intracranial aneurysms ${ }^{15}$ and breast cancer. ${ }^{16}$ However, there is a competing relationship between sensitivity and specificity for a given diagnostic test. Where prevalence is low, very often the number of false positive screening tests far outweighs the population benefit of identifying pre-symptomatic disease. More patients may be harmed by the necessity of invasive investigation procedures (e.g., biopsy) or needless worry than saved by the identification and effective treatment of the identified pathology. The same principles can be applied to the identification of incidental but potentially pathological findings detected with research imaging procedures. Most findings will be benign, but because they have been identified, they require investigation and management.

Investigators have, we believe, an ethical imperative to be transparent in their process of managing incidental findings and disclose to participants whether or not a specialist will be reviewing the imaging results. Surveys designed to capture subjects' expectations after participating in neuroimaging studies revealed that the majority ( $84 \%$ ) of volunteers did not expect all research scans to be reviewed by a physician. ${ }^{17}$ However $54 \%$ of volunteers also believed that brain abnormalities would be revealed if they existed, despite a statement to the contrary in the consent form ("investigators for this project are not trained to perform radiological diagnosis, and the scans performed in this study are not optimized to find abnormalities"). ${ }^{17}$ Therefore, the language used to convey information in the consent forms of many studies, which receive ethics board approval at academic institutions, may not be sufficient to prevent "therapeutic misconception" or the assumption of clinical care in a research setting. ${ }^{18}$

This study has several limitations. First, our report is limited by a lack of follow-up on the implications to treatment and management. We were unable to gather this information because this was not the purpose of our study and we did not obtain consent to gather these data. Second, characteristic MS lesions often unblinded the neuro-radiologist to the participant's disease status and therefore bias in the detection of incidental findings cannot be excluded. Finally, as previously stated, we did not include the detection of white matter lesions in routine MR imaging as an incidental finding in our study design. Therefore, there is the possibility that a control subject could harbor MR evidence of white matter lesions, which would not be viewed as a reportable incidental finding. While more than $95 \%$ of MS patients have MR imaging evidence of focal or confluent abnormalities in white matter, their presence alone does not make the diagnosis of MS. ${ }^{7}$ More specifically, characteristic radiological lesions can appear in people without disease symptoms and non-specific white matter cerebral lesions are common in individuals older than 50 years. ${ }^{7}$ In the post-hoc analysis of our study, we assessed conventional MR studies for several features, including number of lesions, evidence of gadolinium enhancement, and evidence of brain parenchymal/ corpus callosum atrophy. None of our control subjects demonstrated evidence of brain atrophy or gadolinium enhancement, and the white matter lesions found in controls were deemed to be non-specific and not suggestive of underlying demyelination. Therefore, we did not notify control subjects who had non-specific white matter lesions detected on MR imaging. There was evidence of white matter lesions, brain atrophy, and gadolinium enhancement in the MR imaging studies obtained in the MS patients in our study that was part and parcel of their established disease. Because this information did not affect their disease status (which was known) or follow-up in the clinical setting, we did not notify MS patients about the detection of white matter lesions detected on MR imaging. In short, the white matter lesions in these individuals were not incidental, but part of their disease status. 


\section{Conclusion}

Approximately one in every six participants in our study was notified of an incidental MR imaging finding not related to MS or CCSVI. Processes to identify and manage incidental findings are important in designing imaging research studies. A greater awareness for what researchers should anticipate as incidental findings and improved communications with participants will likely stem from a transparent approach to these unexpected results.

\section{ACKNOWLEDGMENTS AND CONTRIBUTIONS}

The authors thank the patients and controls for participating in the study. RF is a Canada Research Chair. The support of the Hotchkiss Brain Institute Neuroimaging Research Unit and the Hopewell Professorship in Brain Imaging is acknowledged. The study was funded by the Multiple Sclerosis Society of Canada.

Design and conduct of the study (FC, MDH); data collection (FC, JT, JNS, JM, VC, DL, DB); study supervision (FC, MDH, $\mathrm{MG}, \mathrm{RF}$ ); analysis and interpretation of the data (FC, MDH, JNS, JM, JT, VC, RF, MG, DL, DB, WJD, JKM, JMB); and preparation (JT, MDH) and critical revision of the manuscript (FC, JNS, JM, VC, RF, MG, DL, DB, WJD, JKM, JMB).

\section{FuNDING}

This work was supported by the Multiple Sclerosis Society of Canada.

\section{CONFLICT OF INTEREST}

$\mathrm{RF}$ is an inventor of the 3D TRICKS approach used in this study. FC has received honoraria for advisory board participation from Questor; and as an educational speaker for EMD Serono. All other authors have no disclosures.

\section{REFERENCES}

1. Palmour N, Affleck W, Bell E, et al. Informed consent for MRI and fMRI research: analysis of a sample of Canadian consent documents. BMC Med Ethics. 2011;12:1.
2. Illes J, Rosen AC, Huang L, et al. Ethical consideration of incidental findings on adult brain MRI in research. Neurology. 2004;62(6):888-90.

3. Katzman GL, Dagher AP, Patronas NJ. Incidental findings on brain magnetic resonance imaging from 1000 asymptomatic volunteers. JAMA. 1999;282(1):36-9.

4. Shoemaker JM, Holdworth MT, Aine C, et al. A practical approach to incidental findings in neuroimaging research. Neurology. 2011;77(24):2123-7.

5. Vernooij MW, Ikram MA, Tanghe HL, et al. Incidental findings on brain MRI in the general population. $\mathrm{N}$ Engl $\mathrm{J}$ Med. 2007;357(18):1821-8

6. Lublin FD, Reingold SC. Defining the clinical course of multiple sclerosis: results of an international survey. National Multiple Sclerosis Society (USA) Advisory Committee on Clinical Trials of New Agents in Multiple Sclerosis. Neurology. 1996;46(4): 907-11.

7. Compston A, Coles A. Multiple sclerosis. Lancet. 2008;372(9648): 1502-17.

8. Mancuso AA. Oh \#*\$\%\#! Another pesky incidental thyroid nodule! AJNR Am J Neuroradiol. 2005;26(10):2444-5.

9. Raymond J, Long H. Thyroid nodules and other incidentalomas: we must search for evidence and clarify our ethical priorities. AJNR Am J Neuroradiol. 2006;27(6):1163-4.

10. Illes J, Kirschen MP, Karetsky K, et al. Discovery and disclosure of incidental findings in neuroimaging research. J Magn Reson Imaging. 2004;20(5):743-7.

11. Racine E, Northoff G, Menon RS, Kimmelman J, Illes J. A Canadian perspective on ethics review and neuroimaging: tensions and solutions. Can J Neurol Sci. 2011;38(4):572-9.

12. Illes J, Kirschen MP, Edwards E, et al. Ethics: incidental findings in brain imaging research. Science. 2006;311(5762):783-4.

13. Almond B. Genetic profiling of newborns: ethical and social issues. Nat Rev Genet. 2006;7(1):67-71.

14. Shickle D, Chadwick R. The ethics of screening: is 'screeningitis' an incurable disease? J Med Ethics. 1994;20(1):12-8.

15. Rinkel GJ. Intracranial aneurysm screening: indications and advice for practice. Lancet Neurol. 2005;4(2):122-8.

16. Kalager M, Zelen M, Langmark F, et al. Effect of screening mammography on breast-cancer mortality in Norway. N Engl J Med. 2010;363(13):1203-10.

17. Kirschen MP, Jaworska A, Illes J. Subjects' expectations in neuroimaging research. J Magn Reson Imaging. 2006;23(2):205-9.

18. Appelbaum PS. Clarifying the ethics of clinical research: a path toward avoiding the therapeutic misconception. Am J Bioeth. 2002;2(2):22-3.

\section{Supplemental Table: MR Imaging Acquisition Parameters}

\begin{tabular}{|c|c|}
\hline FLAIR & $\begin{array}{l}\text { Oblique axial, } 2 \mathrm{D}, \mathrm{TR} / \mathrm{TE}=8000 \mathrm{~ms} / 125 \mathrm{~ms}, \mathrm{TI}=2250 \mathrm{~ms} \\
24 \mathrm{~cm} \times 24 \mathrm{~cm}, 288 \times 224 \text { acquisition matrix, } 48-3 \mathrm{~mm} \text { slices }\end{array}$ \\
\hline FRFSE & $\begin{array}{l}\text { Oblique axial, } 2 \mathrm{D}, \mathrm{TR} / \mathrm{TE}=5591 \mathrm{~ms} / 100 \mathrm{~ms} \\
24 \mathrm{~cm} \times 19.2 \mathrm{~cm}, 512 \times 448,48-3 \mathrm{~mm} \text { slices }\end{array}$ \\
\hline SWAN & $\begin{array}{l}\text { Axial, 3D, flip/TR/TE }=15^{\circ} / 37.2 \mathrm{~ms} / 23 \mathrm{~ms} \\
24 \mathrm{~cm} \times 16.8 \mathrm{~cm}, 320 \times 224,80 \times 2 \mathrm{~mm} \text { partitions, zero-filled to } 160 \text { partitions }\end{array}$ \\
\hline TRICKS & $\begin{array}{l}\text { Sagittal, } 3 \mathrm{D}, \mathrm{flip} / \mathrm{TR} / \mathrm{TE}=15^{\circ} / 2.7 \mathrm{~ms} / 1.0 \mathrm{~ms} \\
40 \mathrm{~cm} \times 24 \mathrm{~cm}, 320 \times 256,62 \times 2.4 \mathrm{~mm} \text { partitions, zero-filled to } 124 \text { partitions } \\
23 \text { temporal phase reconstructed, } 3.46 \mathrm{~s} \text { per phase }\end{array}$ \\
\hline FSPGR & $\begin{array}{l}\text { Sagittal, 3D, flip/TR/TE }=15^{\circ} / 6.4 \mathrm{~ms} / 2.3 \mathrm{~ms} \\
40 \mathrm{~cm} \times 24 \mathrm{~cm}, 320 \times 320,196 \times 1 \mathrm{~mm} \text { partitions, zero-filed to } 392 \text { partitions }\end{array}$ \\
\hline PC (Head) & $\begin{array}{l}\text { Oblique, } 2 \mathrm{D} \text {, flip } / \mathrm{TR} / \mathrm{TE}=20^{\circ} / 33 \mathrm{~ms} / 7.1 \mathrm{~ms} ; 24 \mathrm{~cm} \times 24 \mathrm{~cm}, 256 \times 192,1-4 \mathrm{~mm} \text { slice } \\
\text { Velocity encoding }=40 \mathrm{~cm} / \mathrm{s} \text {, cine acquisition }-16 \text { cardiac phases reconstructed }\end{array}$ \\
\hline PC (Neck) & $\begin{array}{l}\text { Oblique, } 2 \mathrm{D} \text {, flip } / \mathrm{TR} / \mathrm{TE}=20^{\circ} / 27 \mathrm{~ms} / 7.7 \mathrm{~ms} ; 24 \mathrm{~cm} \times 24 \mathrm{~cm}, 256 \times 192,1-4 \mathrm{~mm} \text { slice } \\
\text { Velocity encoding }=15 \mathrm{~cm} / \mathrm{s} \text {, cine acquisition }-16 \text { cardiac phases reconstructed }\end{array}$ \\
\hline
\end{tabular}

FLAIR, fluid attenuate inversion recovery; FRFSE, fast relaxation fast spin echo sequence; FSPGR, sagittal spoiled-gradient echo; PC, phase contrast; SWAN, star weighted angiography; TE, echo time; TR, repetition time; TRICKS, timeresolved imaging of contrast kinetics 